\title{
Multidisciplinary Design Optimization Framework for the Pre Design Stage
}

\author{
M. Guenov †• P. Fantini • L. Balachandran • \\ J. Maginot • M. Padulo $\bullet$ M. Nunez
}

\begin{abstract}
Presented is a novel framework for performing flexible computational design studies at preliminary design stage. It incorporates a workflow management device (WMD) and a number of advanced numerical treatments, including multi-objective optimization, sensitivity analysis and uncertainty management with emphasis on design robustness. The WMD enables the designer to build, understand, manipulate and share complex processes and studies. Results obtained after applying the WMD on various test cases, showed a significant reduction of the iterations required for the convergence of the computational system. The tests results also demonstrated the capabilities of the advanced treatments as follows:

- The novel procedure for global multi-objective optimization has the unique ability to generate well-distributed Pareto points on both local and global Pareto fronts simultaneously.

- The global sensitivity analysis procedure is able to identify input variables whose range of variation does not have significant effect on the objectives and constraints. It was demonstrated that fixing such variables can greatly reduce the computational time while retaining a satisfactory quality of the resulting Pareto front.

- The novel derivative-free method for uncertainty propagation, which was proposed for enabling multi-objective robust optimization, delivers a higher accuracy compared to the one based on function linearization, without altering significantly the cost of the single optimization step.
\end{abstract}

The work demonstrated for the first time that such capabilities can be used in a coordinated way to enhance the efficiency of the computational process and the effectiveness of the decision making at preliminary design stage.

Keywords Design Computational Workflow, Multidisciplinary Design Optimisation, Multiobjective Optimisation, Robust Optimisation, Sensitivity Analysis, Uncertainty Management.

\author{
M. Guenov († $\left.\dagger^{\text {Corresponding Author }}\right) \bullet$ L. Balachandran • J. Maginot • M. Padulo • M. Nunez \\ Advanced Engineering Design Group, \\ Dept. of Aerospace Engineering, Cranfield University, \\ MK43 0AL,Cranfield, Bedfordshire,UK \\ e-mail: m.d.guenov@cranfield.ac.uk \\ P. Fantini \\ Aircraft Research Association Ltd., \\ MK41 7PF, Manton Lane, Bedford, UK \\ e-mail: pfantini@ara.co.uk
}




\section{Introduction}

Our initial research, as part of the EU FP6 VIVACE project, indicated that although involving a lot of talent and producing great results, the existing pre design processes can be significantly improved. For example, relevant process information is currently spread in tools, manuals, brains and sites and in many cases the procedural coding incorporates 'hardwired' assumptions from other disciplines (e.g., weight estimation procedure incorporating assumptions on aerodynamic loading). Thus, the result of a design study may depend on the way it was produced. On the other hand, innovation may be restrained by the confinement to known cases since the design starts from existing configurations, implicit in the computational code. Industrial need was therefore identified for a new approach, allowing to merge numeric and geometric design and to facilitate a component-driven modularisation of the disciplines.

In this context, Cranfield University had the task to research and develop a prototype workflow management device (WMD) enabling a simple formalism and easily understandable description of the computational workflow which would allow to store not only the results, but also the way these were computed. This means that the computational workflow should be stored in an executable format and also be editable by the user in order to develop dynamic solutions. The WMD should be capable of dynamic assembly of hierarchical computational processes from other processes and/or from atomic models (i.e., equations or compiled codes referred to as black boxes). In addition, the WMD should enable the dynamic application of relevant treatments to the computational processes, such as multi-objective optimization, sensitivity analysis and uncertainty management. The realisation of these treatments includes distinct research contribution which is also summarised in this paper.

The relation of this work to the classical notion of MDO, i.e., optimization of a system consisting of coupled disciplines, becomes apparent when considering the objective of pre design, which is to define the characteristics on an aircraft given its properties. That is, to determine the design parameters, given performance and operational parameters derived from stakeholders' requirements, or to modify existing aircraft for the satisfaction of a different or a stretched requirement. In any case a workflow consisting of hundreds of models (black boxes) and thousands of variables needs to be assembled and "(re)wired" every time a variable is added to or removed from the input set. During this process subsets of models may become coupled through shared variables. These coupled or strongly connected components (SCCs) correspond to the 'disciplines' in the classical MDO. The difference is that the pre-design MDO process has to be configured on the fly, with hundreds, albeit low fidelity models.

These problems, as part of the WMD specification are outlined in the next section. The novel Calculation Engine for multi-objective optimization, and in particular, for finding local and global Pareto surfaces is described in section 3. Section 4 discusses sensitivity analysis and in particular, the possibility to identify design variables whose range of variation does not have significant effect on the objectives and constraints. This could significantly reduce the dimensionality of the design space and the computational effort, respectively. Uncertainty management is discussed in section 5 with emphasis on robust multi-objective optimization. In each of these sections appropriate reference to stat of the art in the particular field is made for completeness. The results are presented in section 6 . Finally conclusions are drawn and future work outlined.

\section{Computational workflow management}

The computational process modeller is presented in Fig. 1. A brief summary of the flow chart steps is given below while a detailed description of the associated techniques is presented in Guenov et al. [9], Balachandran et al.[1] and Balachandran [2]. 


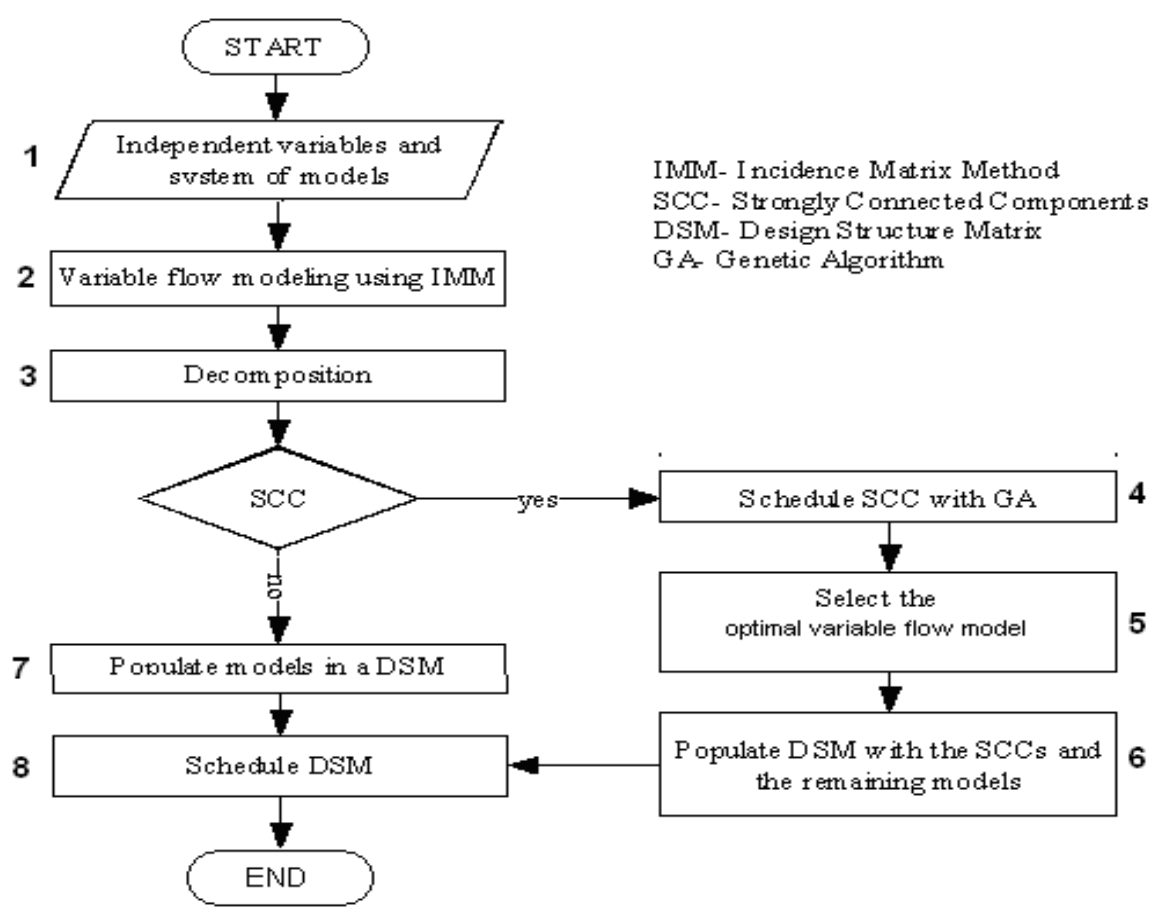

Fig. 1 The computational process modeller

START

Step 1: Initially the designer needs to provide the system of models with a choice of independent (input) variables.

Step 2: Variable flow modelling is performed using the incidence matrix method (IMM) in order to determine the information (data) flow between the models. All feasible variable flow models of the system are explored in this step.

Step 3: Each variable flow model generated is separated into hierarchically decomposable and non-hierarchically decomposable systems of models. Non-hierarchically decomposable systems are also known as strongly connected components (SCC).

Step 4: Given a SCC, its constituent models are rearranged by means of Genetic Algorithm (GA).

Step 5: The selection of the optimal variable flow model is based on the value of objective function which combines criteria such as number of modified models and number and length of the feedback loops.

Step 6: Each of the rearranged SCCs is regarded as a single model and is reintroduced into the DSM along with the remaining models.

Step 7: If SCCs do not exist, then the models are populated directly into a DSM based on data flow obtained from the variable flow model.

Step 8: The DSM is rearranged into a lower triangular matrix based on a graph theoretical algorithm. This rearrangement eliminates the feedback loops and thus the final computational plan of the system is obtained.

END

\section{Multiobjective optimization - generating a well-distributed set of Pareto points}

A number of methods for obtaining an evenly distributed set of Pareto points have been developed in recent years. All of these are based on performing a subdivision of the criterion (objective) space in a set of domains. The optimization problem is then reformulated for each domain, for each of which a Pareto point is generated.

Das and Dennis [5,6] were the first to provide a method for generating well-distributed Pareto points, the Normal-Boundary intersection (NBI) method. Another method is the PP-based method developed by Messac et al. [14] as an extension of the a priori articulation of preference method 
known as the Physical Programming (PP) [17]. The new Normal Constraint (NC) method [15, 16] developed recently also looks very promising.

All of the cited methods have a clear geometrical interpretation, they are all based on the wellknown fact that a Pareto frontier belongs to the boundary of the feasible space towards the minima of the objective functions [18].

The NBI, NC and PP-based methods all follow a similar approach for obtaining well-distributed Pareto points. Anchor points $\boldsymbol{a}_{i}\{\mathrm{i}=1, \ldots, \mathrm{M}\}$ [13], which are the minima relative to each of the objective, are obtained first. Subsequently a number of evenly distributed points belonging to the criterion space, the utopia plane points $\mathbf{p}$, are obtained as linear combinations of the $M$ anchor points [15]. These are used as reference points allowing the reformulation of the optimization problem. Finally, for each utopia plane point $\mathbf{p}$ an optimization is executed in order to obtain a Pareto point. Each of these three steps is fundamental in order to obtain a complete representation of the Pareto frontier.

When dealing with multiobjective optimization problems, for which the number of objectives is greater than two, a peripheral region exists. The peripheral region is that region of the criterion space, for which the orthogonal projection of the Pareto frontier on the utopia plane is external to the polygon spanned by the $M$ anchor points $\boldsymbol{a}_{\boldsymbol{i}}[5,16]$. In their work Das and Dennis limit the importance of the peripheral region, stating that such region will be of no interest to the designer, while Messac and Mattson are interested in obtaining a complete representation of the Pareto frontier.

Even though Das and Dennis believe that such points might be of no interest for the designer, it must be noted that the size of the peripheral region of the Pareto frontier is dependent on the positioning of the anchor points and can be significant. This was demonstrated by Fantini [7] who after analysing the existing methods defined of a set of requirements for an effective method for generating well-distributed Pareto. These requirements state that the reformulation should avoid the introduction of local minima while minimizing the possibility of the optimiser to fail. A unique optimization should be performed for each of the sub-problems in order to minimize the risk of failure. Furthermore, the method should be independent of the number of objectives.

The attempt [7] at improving the reformulation of the optimization problems, in order to remove the limitations associated with the existing methods has followed three consecutive stages. The result of the first one is the modified PP-based method, developed through modification of the PPbased method $[27,10]$. The second one has taken advantage of the experience gained from the development of the first one and has led to the development of the Double Hyper-cone Boundary Intersection (DHCBI) method [7, 8]. The third and latest method developed is the NC+ method. The methods follow the tracks laid by the NBI, PP-based and NC methods, combining the various approaches and the knowledge gained from them. Its formulation is as follows:

subject to $K$ inequality constraints:

$P$ equality constraints:

subject to the additional $M-1$ constraints:

and subject to the additional constraints:

with:

$$
\begin{array}{ll}
\min \boldsymbol{f}(\boldsymbol{x}) & \\
g_{k}(\boldsymbol{x}) \leq 0, & k=1,2, \ldots, K \\
h_{p}(\boldsymbol{x})=0, & p=1,2, \ldots, P \\
\boldsymbol{v}_{\boldsymbol{j}}\left(\boldsymbol{p}_{\boldsymbol{i}}-\boldsymbol{f}\right) \leq 0, & \forall \boldsymbol{j} \in\{1,2, \ldots, M\}, j \neq l \\
\boldsymbol{v}_{\boldsymbol{j}}\left(\boldsymbol{f}-\boldsymbol{p}_{\boldsymbol{i}}-n_{c} \boldsymbol{v}_{\boldsymbol{l}} /\left\|\boldsymbol{v}_{\boldsymbol{l}}\right\|\right) \leq 0, \\
\boldsymbol{x}_{L} \leq \boldsymbol{x} \leq \boldsymbol{x}_{U},
\end{array}
$$

where $\boldsymbol{v}_{\boldsymbol{j}}=\left(l_{j} / l_{i}\right) \boldsymbol{e}_{\boldsymbol{l}}-\boldsymbol{e}_{\boldsymbol{j}}$ for $j \neq l, \boldsymbol{v}_{\boldsymbol{l}}=\boldsymbol{m} \boldsymbol{I} \boldsymbol{e}_{\boldsymbol{l}} / l_{l}-\boldsymbol{m}, \boldsymbol{e}_{\boldsymbol{j}} \forall \boldsymbol{j}$ are the base vectors of the coordinate system, $\boldsymbol{I}$ is the unit vector orthogonal to the utopia plane, $n_{c}$ is a fraction of the Euclidean distance between two contiguous utopia plane points, $\boldsymbol{m} \in \mathbb{R}^{M}$ is a vector such that $m_{i}=1 \forall i$ and $m_{l}=0$ and $\boldsymbol{x}_{L}, \boldsymbol{x}_{U}$ are the lower and upper bounds for the input variables, respectively.

The formulation is similar to the NC method, where $M$ - 1 constraints are used for building $M$ - 1 hyper-planes which confine the solution to a region of the criterion space. Although in the NC method these constraints are dependent on the anchor points, in the NC+ method they are build with respect to the coordinate system.

For the solution to belong to the line orthogonal to the utopia plane, passing through a particular utopia plane point, all hyper-planes need to be orthogonal to the utopia plane and have to intersect in the utopia plane point. In the NC+ method, the hyper-planes are determined with respect to the coordinate system in such a way that the orthogonality condition is always enforced [7]. In order to minimize the possibility of obtaining solutions not belonging to the line orthogonal to the utopia 
plane, passing through utopia plane point $\boldsymbol{p}_{\boldsymbol{i}}$, an additional constraint is added. Constraint $l$ is built in order to reduce the size of the feasible region, confining the solution in the proximity of the line passing through utopia plane point $\boldsymbol{p}_{\boldsymbol{i}}$. As for the other $M-1$ constraints, constraint $l$ defines a hyper-plane orthogonal to the utopia plane, but positioned at a distance $n_{c}$ in the direction of vector $\boldsymbol{v}_{\boldsymbol{l}}$. Further details on the method can be found in Fantini [7].

\section{Sensitivity analysis}

Sensitivity analysis (SA) is the study of how changes in the outputs of a complex model can be apportioned qualitatively or quantitatively to variations in the different inputs. Unfortunately, information on models used to describe a complex system cannot be obtained analytically because the internal mechanisms are not known. This is particularly true in MDO since complicated coupling between disciplines must be taken into account. In such a situation, only numerical results can be obtained. A way to get a better understanding of the model is to perform a samplebased sensitivity analysis. In such a procedure, the model is executed repeatedly for a set of input values. Saltelli [22] describes in detail the steps necessary to perform a sample-based sensitivity analysis as summarised in Fig. 2.

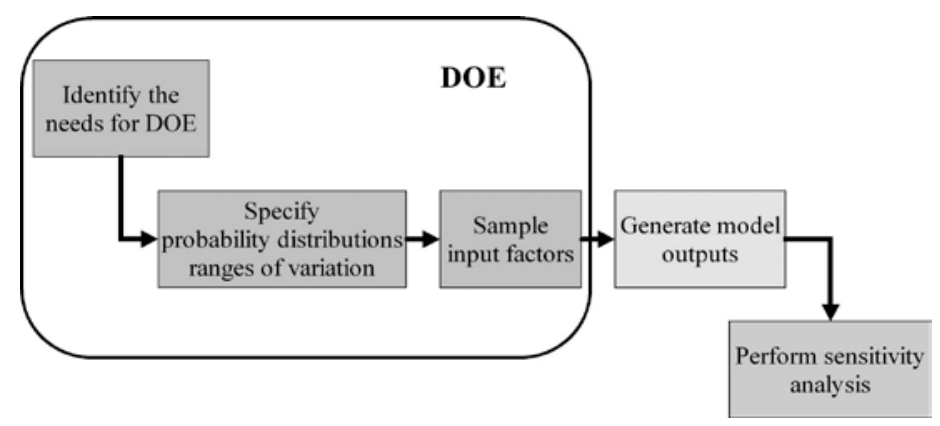

Fig. 2 Typical sensitivity analysis procedure

\subsection{Variance-based methods}

When implementation of sensitivity analysis is concerned, many different approaches can be followed. We have chosen Variance-based methods (VBM) since these are rigorous and theoretically sound approaches for global sensitivity calculation [23] exhibiting desirable properties for sensitivity analysis of complex models.

VBM provide quantitative information on the influence of each input factor to help the designer identify the most influential variables, on which the computational effort can be concentrated, and the variables with negligible effects which can be discarded or frozen to a specific value.

Variance-based approaches for sensitivity analysis decompose the output variance into partial variances of increasing dimensionality.

$$
V(Y)=\sum_{i} V_{i}+\sum_{i \neq j} V_{i j}+\ldots+V_{1,2,3, \ldots, k}
$$

In the decomposition of the variance, the term $V_{i j}$ is the interaction effect between $x_{i}$ and $x_{j}$. $V_{i j}$ represents the part of the output variation due to input parameters $x_{i}$ and $x_{j}$ which cannot be explained by the sum of the first order effects of parameter $x_{i}$ and $x_{j}$. Similar considerations can be made for higher order terms.

The two coefficients of main interest for sensitivity are:

- $\quad$ The main effect index which gives the first-order contribution of $X_{i}$ to the output response.

- $\quad$ The total effect index which gives the total contribution of $X_{i}$ to the output response.

For each input, the difference between its total effect and its main effect gives an indication of the importance the contribution to the output due to interactions with other inputs. Therefore, both the 
main effect indices and total effect indices are necessary to obtain information about the nonadditivity of the model and on the relative importance of variable interactions.

Two main methods, Fourier Amplitude Sensitivity Test (FAST) [4, 24, 25, 26], have been developed to compute the different terms of the variance decomposition and both main and total effect indices.

\subsection{Reducing the dimensionality of the optimization problem}

In the context of MDO, we assume that the model, which evaluates the objectives and constraints of the optimization problem, can be represented as a black-box. This stands for the fact that the designer is unaware of the complex internal mechanisms of the model, which relate objectives and constraints to the input variables. The methodology developed in this general case can then be readily implemented for a more specific purpose. In the context of deterministic optimization, it is also assumed that all variables have uniform distribution over their ranges $\left[\boldsymbol{x}_{L}, \boldsymbol{x}_{U}\right]$. In this approach, it is proposed to use VBM to evaluate the global sensitivity indices of each input with respect to all outputs. This allows to quantify the effect of the variations of the inputs on the outputs variance. Both main and total sensitivity indices are calculated. The main and total effects represent the minimum and maximum expected reduction of the output variance if the input is fixed to a specific value. Therefore, freezing an input variable with a negligible total sensitivity index will not affect the output variance.

It is proposed to remove from the original optimization formulation a variable with a negligible effect on all objectives and constraints. When more than one variable is non-significant, the designer should consider these as a group of variables and see whether the sensitivity of the group is still below the significance threshold [11, 12]. Typically, this would imply performing another sensitivity analysis and re-sampling the design space. Such procedure could be very expensive. Instead, it is recommended to make sure that the sum of the group's total indices remains below a particular threshold value. Without loss of generality, let us assume that the first $R$ variables are non-significant to all objectives and constraints. The problem can be reformulated as follows:

$$
\begin{array}{lll}
\text { subject to K inequality constraints: } & g_{k}\left(\boldsymbol{x}_{\text {red }}\right) \leq 0, & k=1,2, \ldots, K \\
\text { and P equality constraints: } & h_{p}\left(\boldsymbol{x}_{\text {red }}\right)=0, & p=1,2, \ldots, P \\
\text { with: } & \boldsymbol{x}_{\text {red }_{L}} \leq \boldsymbol{x}_{\text {red }} \leq \boldsymbol{x}_{\text {red }_{U}}, &
\end{array}
$$

where $\boldsymbol{x}_{\text {red }}=\left(x_{R+1}, \ldots, x_{N}\right)$. All variables with negligible effect are fixed to a value $x_{m}=x_{m}{ }^{*}$ for $m=$ $1, \ldots, R$.

The approach is presented in the case of a multi-criteria optimization (see section 6.3), but the same considerations can be made when a single objective is optimised.

\section{Design robustness}

A constrained robust optimization (RO) strategy can be thought of as made up of three main parts. The first stage consists of identifying, qualifying and quantifying the sources of uncertainty associated with the design input and the analysis modules. This is usually done by means of stochastic models. The second phase consists of propagating the uncertainty through the analysis system, to adequately model the probabilistic behaviour of the objective functions and constraints. The obtained probabilistic quantities are hence used in the third stage of the process, during which the optimization is performed.

In $\mathrm{RO}$, the probabilistic state can be defined in terms of expectation and variance of the deterministic objectives and constraints. A single-objective deterministic optimization problem turns then into a multi-objective robust problem if the two statistical moments are thought of as representing, two conflicting objectives such as a suitable average of the system performance and its sensitivity to unforeseen variations, respectively [3]. Several approaches have been developed to adequately accommodate this issue, ranging from the weighted sum method to physical programming [21]. When the considered deterministic problem is multi-objective, multiple system 
performance metrics have to be traded-off [19]. In our case, the hypothesis of independence of the deterministic objectives is adopted. This leads to formulating the original bi-objective problem as a four objective one in which robustness is sought for by minimizing the objectives' variances, while expectations of the performance measures are optimized according to their physical meanings. To maintain design feasibility with a specific level of confidence, given the prescribed input uncertainty, inequality constraints of the form $g_{k}(x) \leq 0$ take the following form:

$$
\mu_{g_{k}}(x)+t_{g_{k}} \sigma_{g_{k}}(x) \leq 0
$$

The coefficient $t_{g_{k}}=\Phi^{-1}\left(P\left(g_{k} \leq 0\right)\right)$ guarantees a prescribed level of probability $P$ of constraint satisfaction, where $\Phi^{-1}$ is the inverse of the normal cumulative distribution function. It is to be noted that this formulation is only approximate for non-normal constraint functions. Eq. (3) can thus be extended as follows:

$$
\begin{array}{ll}
\text { subject to the inequality constraints: } \quad & G_{k}=\mu_{g_{k}}(x)+t_{g_{k}} \sigma_{g_{k}}(x) \leq 0, \quad k=1,2, \ldots, K \\
& \boldsymbol{x} \boldsymbol{q}+t_{\boldsymbol{x}} \boldsymbol{\mu}_{\boldsymbol{x}} \leq \boldsymbol{x}_{\boldsymbol{x}} \leq \boldsymbol{\sigma}-t_{\boldsymbol{x}} \quad \boldsymbol{x} .
\end{array}
$$

The way in which mean and variance of each objective and constraint function are obtained starting from the knowledge of the uncertainty affecting $\boldsymbol{x}$ turns out to be crucial both for the efficiency and the accuracy of the whole RO approach. If all the variables are continuous, the first two moments of $y=f(x)$ are:

$$
\begin{aligned}
& \mu_{f}\left(x \xi=\int_{-\infty}^{+\infty} f \xi() p_{X}() d,\right. \\
& \left.\sigma_{f}^{2}(x) \xi \int_{-\infty}^{+\infty} \xi(\xi)-\mu_{f}(x)\right\}^{2} p_{X}() d,
\end{aligned}
$$

where $p_{X}$ is the joint probability density function corresponding to the distributions modelling the uncertainty of the input variables, and is supposed not to depend on the design point $\boldsymbol{x}$. Since a closed-form solution of these integrals can be obtained only in a few cases of practical interest, uncertainty propagation is usually performed in an approximate fashion. As pointed out in Padulo et al. [20], an attractive compromise between cost and accuracy of the propagation phase is offered by reduced quadrature methods, such as the Sigma-Point (SP) approach. This method gives mean and variance as follows:

$$
\begin{aligned}
& \mu_{f}=W_{0} f\left(x_{0}\right)+\sum_{p=1}^{n} W_{p}\left[f\left(x_{p+}\right)+f\left(x_{p^{-}}\right)\right], \\
& \sigma_{f}^{2}=\frac{1}{2} \sum_{p=1}^{n}\left\{W_{p}\left[f\left(x_{p_{+}}\right)-f\left(x_{p_{-}}\right)\right]^{2}+\left(W_{p}-2 W_{p}^{2}\right)\left[f\left(x_{p+}\right)+f\left(x_{p_{-}}\right)-2 f\left(x_{0}\right)\right]^{2}\right\} .
\end{aligned}
$$

The weights are chosen as follows:

$$
\begin{aligned}
& W_{0}=\frac{h_{s p}^{2}-n}{h_{s p}^{2}}, \\
& W_{p}=\frac{1}{2 h_{s p}^{2}}, \text { for } 1 \leq p \leq n .
\end{aligned}
$$

The sampling points are:

$$
\begin{aligned}
& \boldsymbol{x}_{0}=\mu_{\boldsymbol{x}}, \\
& \boldsymbol{x}_{p \pm}=\mu_{\boldsymbol{x}} \pm h_{p} \sigma_{\boldsymbol{x}_{p}} \boldsymbol{e}_{p},
\end{aligned}
$$


where $\boldsymbol{e}_{p}$ is the $p^{\text {th }}$ column of the identity matrix of size $n$ and $h_{p}$ is equal to the square root of the kurtosis of the $p^{\text {th }}$ design variable distribution. The accuracy of the SP method, in particular for the mean estimate, is higher with respect to the largely adopted first order Taylor based method of moments (MM). However, it requires only $2 n+1$ function evaluations for each analysis, which is equal to the cost of linearization if function gradients are approximated by centered finite differences. When the function performing the system analysis is differentiable, this technique can be efficiently used in gradient-based optimization; the computational cost of a single optimization step in terms of function evaluations is $\propto n^{2}$ by using either the SP method or MM for the propagation phase, if the derivatives are obtained by finite differencing. If the source code of the analysis system is available and $\mathrm{AD}$ can be deployed, this cost decreases to $\propto n$ in both cases.

\section{Results}

The results of the tests on the workflow manager and the coordinated application of the above described treatments are presented in this section. The adopted test case is an Ultra Simplified Model of Aircraft (USMAC), which was provided by a major airframe manufacturer, in the context of the European project VIVACE. USMAC can determine performance and sizing at conceptual design level for a short-to-medium range commercial passenger aircraft. The test case contains 97 models and 125 variables.

\subsection{Computational Process Modeller Results}

Synthesis of an optimal computational plan has to be performed before any treatment is applied to the test case. To test the capability of the computational process modeller to generate optimal computational plans for a system with suitably chosen inputs, 23 variables were chosen randomly as independent from the 125 variables set (Twenty three variables are required in this test case to ensure that the system is determined). The computational process modeller generated optimal computational plans. The computational cost of each optimal computational plan was then compared with the cost of its corresponding non-optimal plans. The computational cost comparison was based on the number of calls made to the models of the SCC during solving. The SCCs were solved by applying a fixed point iteration method while the modified models were resolved by applying Gauss-Newton method.

After decomposing the system, thirteen out of the 97 models were identified as strongly connected. There were twelve variable flow models generated for the SCC. However, only four of these produced a converged solution. The non-converged ones were those variable flow models which had a higher number of modified models and feedback numbers. Fig. 3 shows an example of a (converged) final computational sequence.

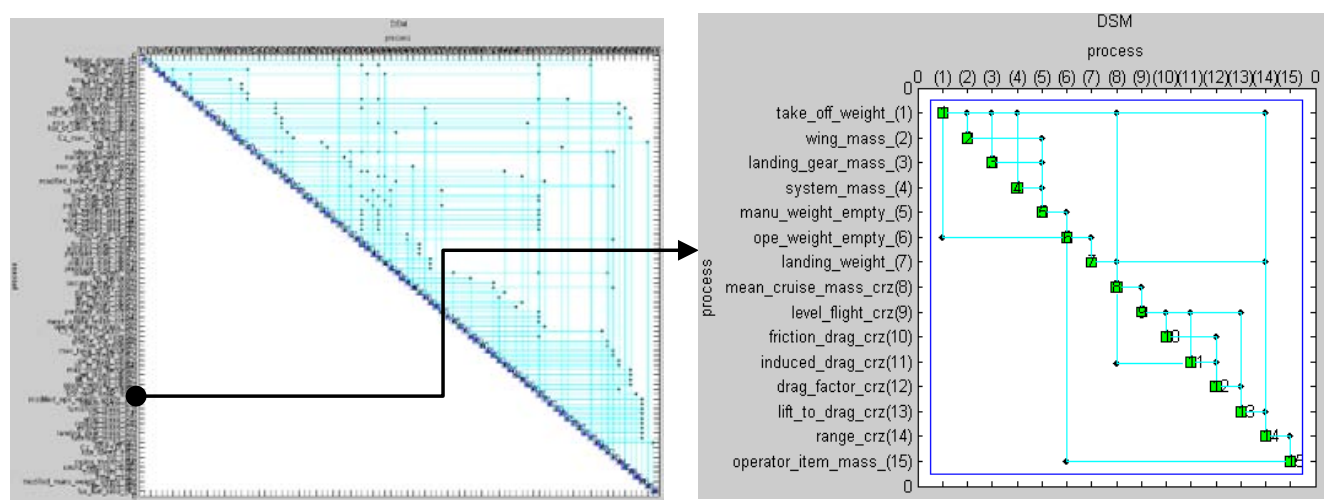

Fig. 3 (a) DSM of the USMAC final computational plan. (b)Design Structure Matrix of the SCC with the shortest feedback length

Table 1 provides details of four converged models and a non-converged solution for comparison. 


\begin{tabular}{cccccc}
\hline $\begin{array}{c}\text { Variable } \\
\text { flow model }\end{array}$ & nFdb & nMm & $\begin{array}{c}\text { Optimal flow } \\
\text { model }\end{array}$ & $\begin{array}{c}\text { Number of calls to the } \\
\text { models in SCC }\end{array}$ & $\begin{array}{c}\% \text { additional } \\
\text { computational }\end{array}$ \\
\hline 1 & 3 & 6 & & 117 & $95 \%$ more \\
2 & 5 & 11 & & 158 & $163 \%$ more \\
3 & 6 & 3 & & 60 & Base \\
4 & 5 & 9 & & 198 & $230 \%$ more \\
5 & 8 & 11 & & Not converged & - \\
\hline \hline
\end{tabular}

Table 1 Details of computational process modelling and solving of SCC

Variable flow model 3 was chosen by the computational process modeller as the optimal one, since it has the smallest number of modified models. It is shown in the table that the selected optimal flow model has the lowest computational cost for the SCC. From Table 1 it is also clear that when the number of modified models increases the computational cost for the SCC also increases. However, for variable flow model 4, even though the number of modified models is less than the one for the flow model 2, it has taken more calls to obtain a converged solution. This discrepancy was observed because the convergence of the SCC was not only depending on the number of modified models and the number of feedback loops, but also on other factors such as the starting (iteration) point for the unknown variables, mutual sensitivity of the switched (input with output) variables of the modified models and possible other factors which are yet to be discovered. Nevertheless, these and many more extensive tests conducted on the process modeller demonstrated that the selections which it made were always amongst the best in terms of SCCs' convergence.

\subsection{Multi-objective optimization results}

The computational process modeller generated an optimal computational plan onto which the NC+ method was executed with the settings presented in Table 2. Five starting points were used in order to determine the multiple local minima. Forty utopia plane points were generated in order to determine the Pareto front. The results obtained are shown in Fig. 4. In addition, an interactive visualization interface has been developed to take into account the traditional aircraft conceptual design approach, which relies heavily on matrix and carpet plots. The proposed visualization tool shows graphically whether a design point meets a set of performance constraints (such as cruise speed, second segment climb rate, direct climb, take-off and landing field lengths), derived from the original constraint set. By clicking on a design point, the corresponding carpet plot is automatically generated. An example of this visualization is shown in Fig. 5. The example also demonstrates that it is possible to find out in which direction the design point under consideration should be moved on the plot to obtain the desired improvements.

\begin{tabular}{|c|c|c|c|c|c|}
\hline \multicolumn{6}{|c|}{ Nomenclature } \\
\hline Npax & \multicolumn{2}{|c|}{ number of passengers } & altcrz & \multicolumn{2}{|c|}{ cruise altitude [ft] } \\
\hline NpaxFront & \multicolumn{2}{|c|}{ number of passengers per row } & Machcrz & \multicolumn{2}{|c|}{ cruise mach } \\
\hline Naisle & \multicolumn{2}{|c|}{ number of aisles } & altto & \multicolumn{2}{|c|}{ takeoff altitude [ft] } \\
\hline FNslst & \multicolumn{2}{|c|}{ sea-level static engine thrust [decaN] } & altapp & \multicolumn{2}{|c|}{ approach altitude [ft] } \\
\hline BPR & \multicolumn{2}{|c|}{ engine bypass ratio } & MTOW & \multicolumn{2}{|c|}{ maximum take-off weight [kg] } \\
\hline ne & \multicolumn{2}{|c|}{ number of engines } & RA & \multicolumn{2}{|c|}{ range $[\mathrm{NM}]$} \\
\hline Awing & \multicolumn{2}{|c|}{ wing area $\left[\mathrm{m}^{2}\right]$} & RAtime & \multicolumn{2}{|c|}{ flight time [h] } \\
\hline span & \multicolumn{2}{|c|}{ wing span $[\mathrm{m}]$} & tofl & \multicolumn{2}{|c|}{ takeoff field length [m] } \\
\hline phi & \multicolumn{2}{|c|}{ wing sweep angle [deg] } & vapp & \multicolumn{2}{|c|}{ approach speed [kts] } \\
\hline tuc & \multicolumn{2}{|c|}{ wing thickness to chord ratio } & vzclb & \multicolumn{2}{|c|}{ climb rate $[\mathrm{ft} / \mathrm{min}]$} \\
\hline MTOW & \multicolumn{2}{|c|}{ maximum take-off weight [kg] } & kfncth & \multicolumn{2}{|c|}{ cruise thrust coefficient } \\
\hline Fuel & \multicolumn{2}{|c|}{ fuel weight [kg] } & Kff & \multicolumn{2}{|c|}{ wing fuselage fuel ratio } \\
\hline \multicolumn{3}{|l|}{ Constant } & \multicolumn{2}{|c|}{ Constraints } & Objectives \\
\hline \multirow{6}{*}{\multicolumn{2}{|c|}{$\begin{array}{l}\text { Npax }=150 \\
\text { NpaxFront = } 6 \\
\text { Naisle = } 1 \\
\text { ne }=2 \\
\text { altcrz }=35000 \mathrm{ft} \\
\text { Machcrz }=0.82\end{array}$}} & FNslst $=[12500,13000]$ & \multirow{6}{*}{\multicolumn{2}{|c|}{$\begin{array}{l}\text { tofl } \leq 2000 \mathrm{~m} \\
\text { vapp } \leq 120 \mathrm{kts} \\
\text { vzclb } \geq 500 \mathrm{ft} / \mathrm{min} \\
\mathrm{kfncth} \leq 1 \\
\mathrm{Kff} \geq 0.75\end{array}$}} & RA [NM] \\
\hline & & Awing $=[152,158] \mathrm{m}^{2}$ & & & to be maximized \\
\hline & & $\operatorname{span}=[30,38] \mathrm{m}$ & & & MTOW [kg] \\
\hline & & phi $=[28,32] \mathrm{deg}$ & & & to be minimized \\
\hline & & tuc $=[0.07,0.1]$ & & & \\
\hline & & Fuel $=[17000,18000] \mathrm{kg}$ & & & \\
\hline
\end{tabular}




\begin{tabular}{|c|c|}
\hline $\begin{array}{l}\text { altto }=0 \mathrm{ft} \\
\text { altapp }=0 \mathrm{ft}\end{array}$ & $\mathrm{BPR}=[6,7]$ \\
\hline
\end{tabular}

Table 2 Set up of the multi-objective optimization problem

Finally, to illustrate how integrating design computation, parametric geometry and configuration can aid the designer in choosing a particular (Pareto) solution, a prototype tool, presented in Fig. 6, was developed. The tool allows for browsing each of the Pareto points while showing the changes to the geometry and indicating (in different colour) which constrains are activate for the particular point.

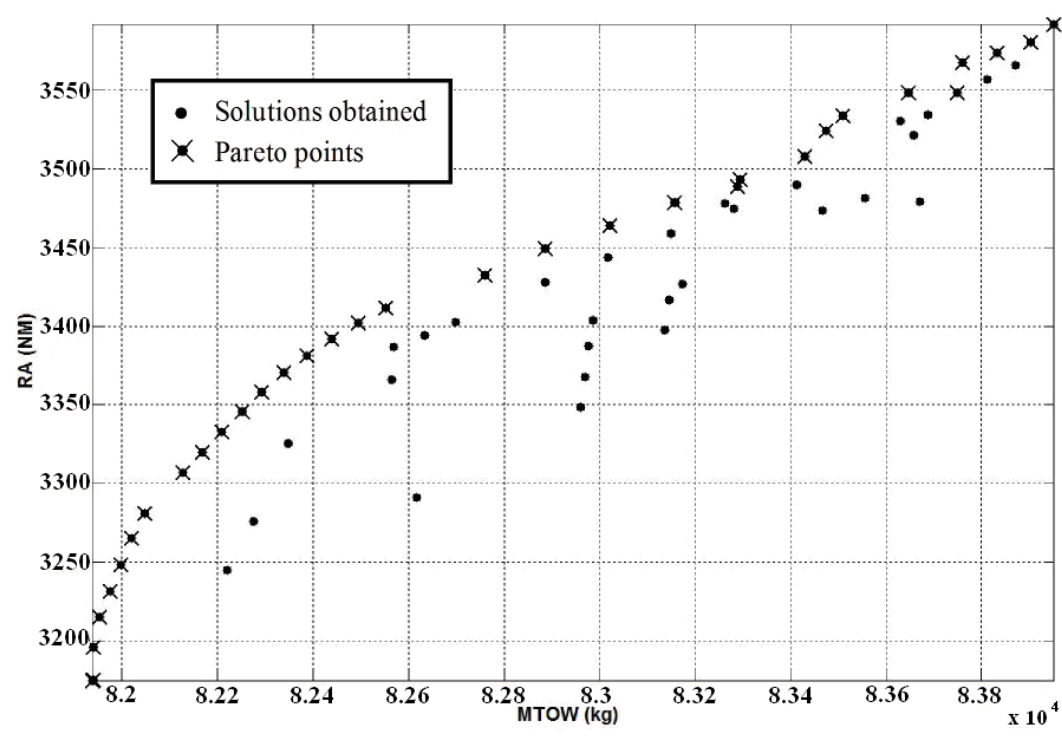

Fig. 4 USMAC test case Pareto front

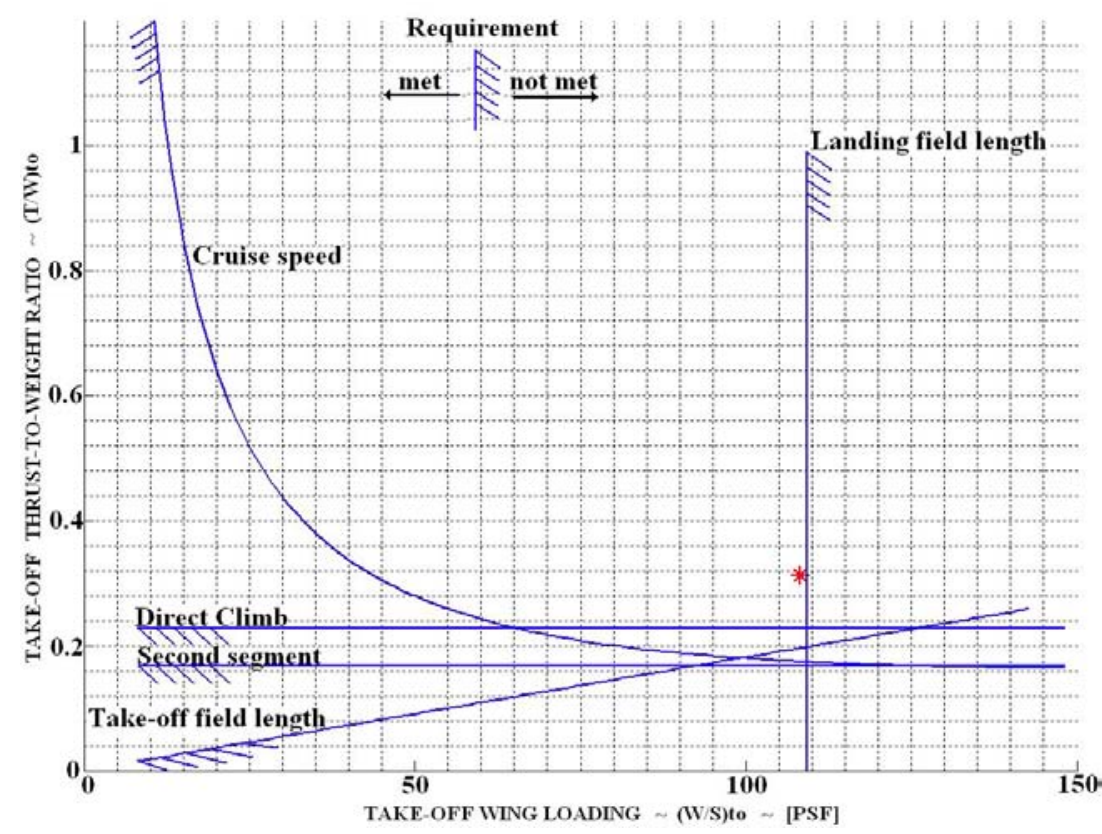

Fig. 5 Example of the carpet plot of a design point 


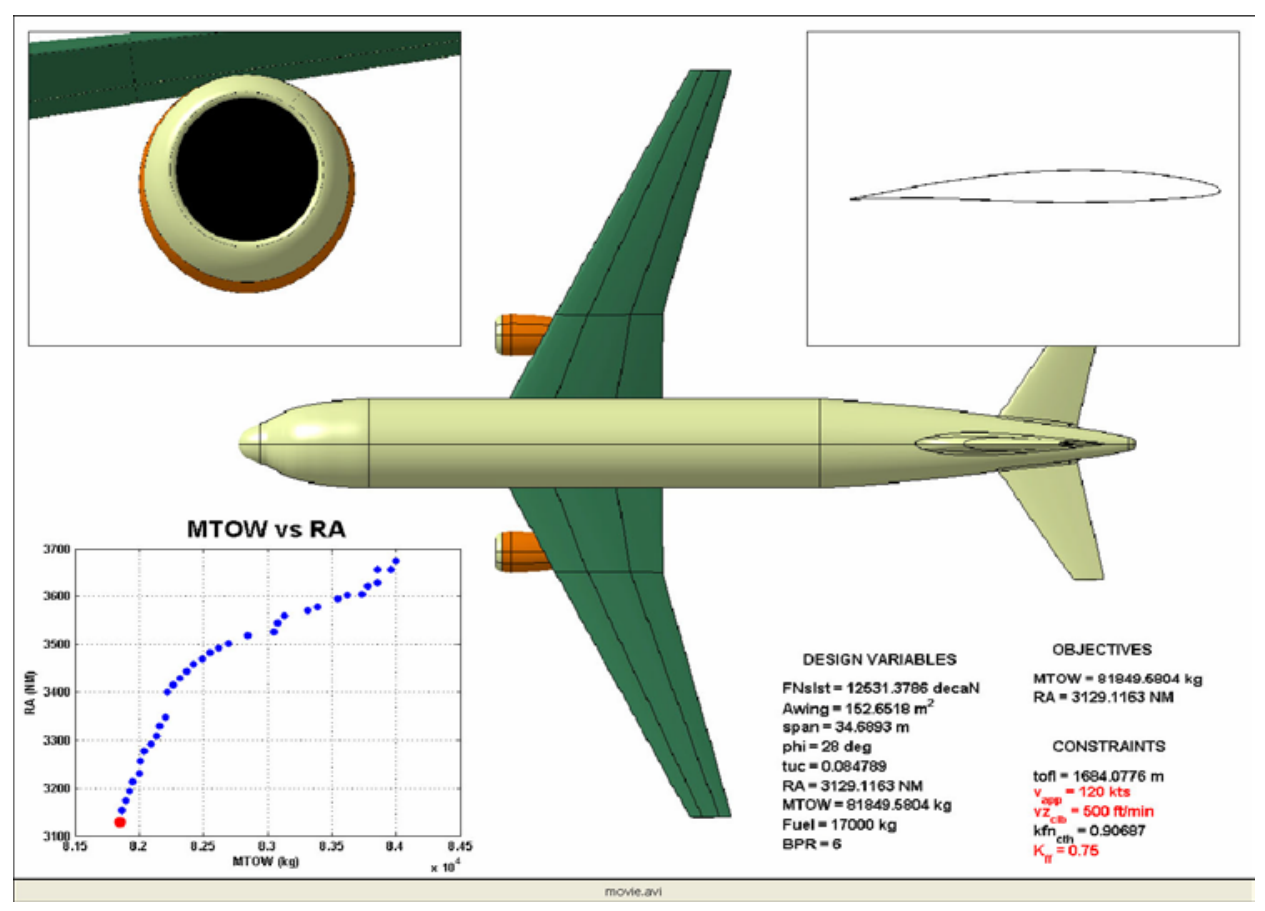

Fig. 6 Visualization of the Pareto set, including geometry and constraint activation

\subsection{Sensitivity analysis results}

For this particular test case and the specific range of variations, it appears that the contributions to the outputs variance due to interactions between inputs are very small. Therefore, one can conclude that contributions to the outputs are entirely due to direct effects. It is appears that FNslst, Span, Wing, tuc and Fuel are the most significant variables to the problem. Variable phi has a smaller effect but cannot be neglected as it affects tofl and vapp. The global indices for BPR for all optimization outputs are almost equal to zero and therefore BPR appears to be negligible for this particular problem. The reduced optimization problem is derived by keeping all variables and fixing BPR to the mean value of its range of variation, i.e. $\mathrm{BPR}=6.5$.

The original Pareto set and the one obtained with the reduced optimization problem are shown in Fig. 7. The two Pareto fronts are very similar in the criterion space which indicates that a similar level of performance can be obtained by only considering variables with a real effect. Freezing BPR to a value resulted in a significant reduction of the computational effort: 16627 function evaluations were needed to obtain the Pareto front compared to 65332 when all variables are considered.

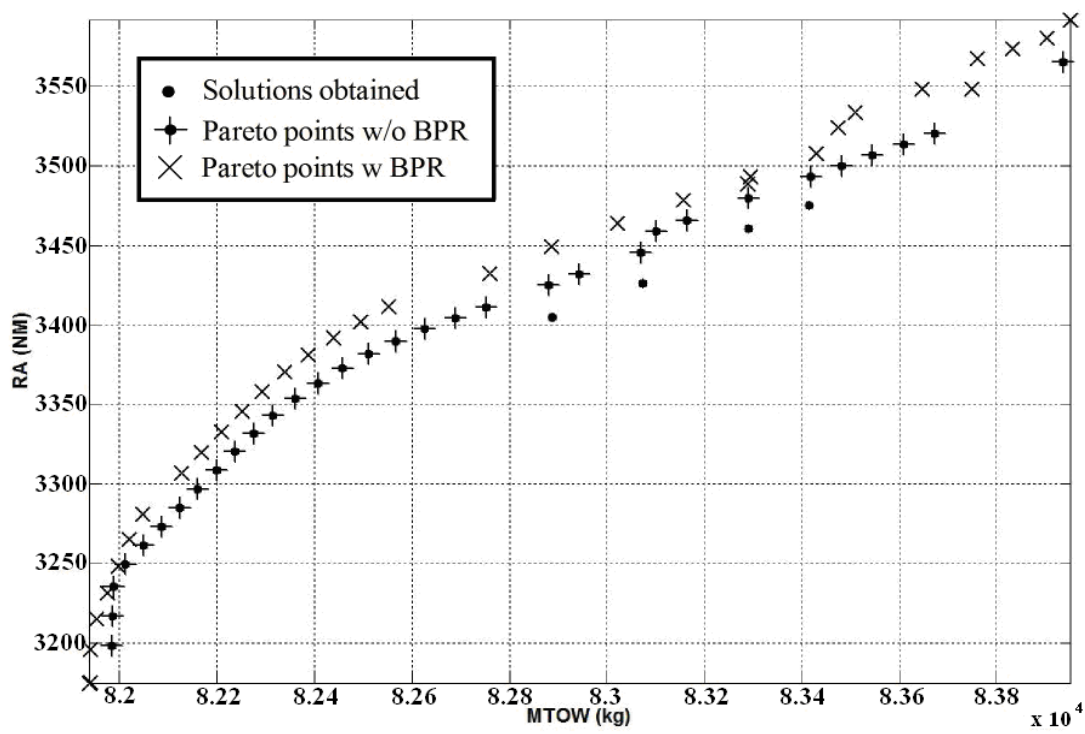

Fig. 7 USMAC Pareto front for full and reduced optimization problem 


\subsection{Robust optimization results}

The robust counterpart of the deterministic optimization problem in Section 0 considers separately, without any a priori assumption on relative weights, mean and variance for the two physical objectives. The problem to be solved is then a 4 objective optimization. The deterministic constraints are transformed into their robust counterpart by adopting $t_{\mathrm{g}}=\mathrm{t}_{\mathrm{x}}=1$ as weighting coefficients in Eq. (3), to impose the robust feasibility with a probability of approximately $84 \%$. The assumed uncertainties of input variables, in terms of standard deviation, are shown in Table 3.

\begin{tabular}{l|ccccccc}
\hline \hline Input variable & FNslst & BPR & Awing & span & phi & tuc & Fuel \\
Standard deviation & 100 decaN & 0.2 & $5 \mathrm{~m}^{2}$ & $0.5 \mathrm{~m}$ & $1 \mathrm{deg}$ & 0.02 & $50 \mathrm{~kg}$ \\
\hline \hline
\end{tabular}

Table 3 Uncertainties of input variables

It is useful to compare the deterministic with the obtained robust Pareto front, by performing an a posteriori uncertainty analysis on the deterministic results in order to obtain mean and variance for each Pareto point. The mean of the objectives for the robust optimization is then superimposed on the same plot (see Fig. 8). The adopted representation is a bidimensional projection of a four dimensional Pareto hypersurface. It has been judged to be appropriate for the problem at hand since the variation of standard deviation for both objectives is negligible with respect to their mean values. This is mainly due to the small input uncertainty considered. Thus the robust optimal solutions turn out to be dominated by the deterministic ones in terms of mean values mainly as a result of the imposed stricter constraints.

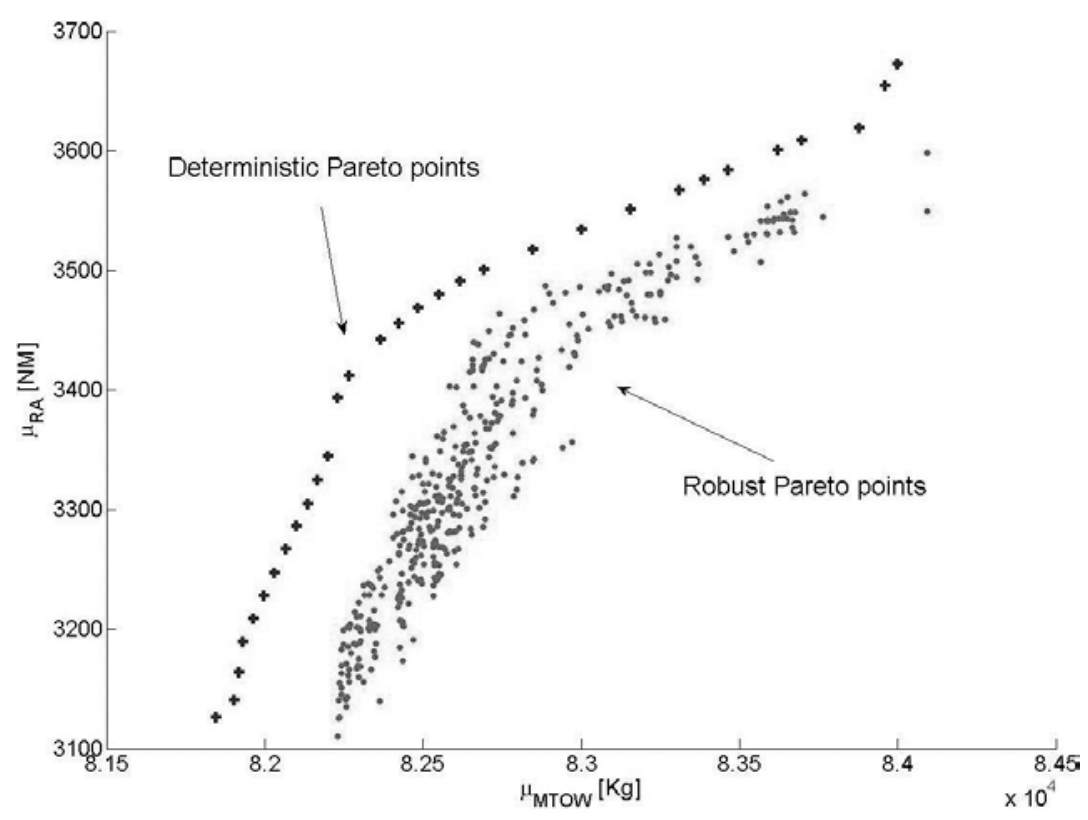

Fig. 8 Comparison of deterministic and robust Pareto fronts

\section{Conclusions}

Presented is a novel computational framework providing the capability for performing flexible design studies at preliminary design stage. It incorporates a workflow management device (WMD) and a number of advanced treatments, including multi-objective optimization, sensitivity analysis and uncertainty management. The WMD enables the designer to build, understand, manipulate and share complex processes and studies. Results obtained after applying the WMD on various test cases, showed a significant reduction of the iterations required for the convergence of the 
computational system. The tests also demonstrated the capabilities of the advanced treatments as follows:

- The novel procedure for global multi-objective optimization has the unique ability to generate well-distributed Pareto points on both local and global Pareto fronts simultaneously;

- $\quad$ The global sensitivity analysis procedure is able to identify input variables whose range of variation does not have significant effect on the objectives and constraints. It was demonstrated that fixing such variables can greatly reduce the computational time while retaining a satisfactory quality of the resulting Pareto front;

- $\quad$ The novel derivative-free method for uncertainty propagation, which was proposed for enabling multi-objective robust optimization, delivers a higher accuracy compared to the one based on function linearization, without altering significantly the cost of the single optimization step.

This work demonstrated for the first time that such capabilities can be used in a coordinated way to enhance the efficiency of the computational process and the effectiveness of the decision making. Future work will be concentrate on further integration of the treatments which will allow their dynamic application as dictated by the computational process. Also, further integration with parametric geometry and configuration tools is planned in order to achieve the ultimate goal of this work, that is, to study unconventional new configurations with a higher level of detail and better risk assessment before proceeding to the next stages of the product development process.

Acknowledgements The research reported in this paper has been carried out within the VIVACE Integrated Project (AIP3 CT-2003-502917) which is partly sponsored by the Sixth Framework Programme of the European Community (2002-2006) under priority 4, “Aeronautics and Space”. The authors wish to thank our industrial partners for the fruitful discussions, constructive criticism and the provision of test cases. Finally we would like to thank the anonymous reviewers for their helpful comments.

\section{References}

1. Balachandran L.K., Fantini, P.F., Guenov, M.D.: Computational Process Management for Aircraft Conceptual Design. 7th AIAA Aviation Technology, Integration and Operations Conference (ATIO), Belfast, September 2007

2. Balachandran L. K.: Computational workflow management for conceptual design of complex systems: An Air-vehicle Design Perspective. PhD Thesis, Cranfield University, Cranfield, UK (2007)

3. Chen, W., Allen, J.: A procedure for robust design: Minimizing variations caused by noise factors and control factors. Journal of Mechanical Design, 118(4), pp. 478-493 (1996)

4. Cukier, R.I., Levine, H.B., Shuler, K.E.: Non-Linear Sensitivity Analysis of Multi-Parameter Model Systems. Journal of Computational Physics, Vol. 26, No. 1, pp. 1-42 (1978)

5. Das, I., Dennis, J.E.: Normal-Boundary Intersection: A New Method for Generating the Pareto Surface in Nonlinear Multicriteria Optimization Problems. SIAM Journal of Optimization, vol. 8, pp. 631-657 (1998)

6. Das, I.: An Improved Technique for Choosing Parameters for Pareto Surface Generation Using Normal-Boundary Intersection. In: Proceedings of the Third World Congress of Structural and Multidisciplinary Optimization WCSMO-3, Buffalo, NY, March 1999

7. Fantini, P.: Effective Multiobjective MDO for Conceptual Design - An Aircraft Design Perspective. PhD Thesis, Cranfield University, Cranfield, UK (2007) 
8. Fantini, P., Balachandran, L.K., Guenov, M.D.: Computational Intelligence in Multi Disciplinary Optimization at Conceptual Design Stage. In: Proceedings of the First International Conference on Multidisciplinary Design Optimization and Applications, Besancon, France, April 2007

9. Guenov, M.D., Libish, Tang, D., Lockett, H.: Computational Design Process Modelling. In: Proceedings of 25th International Council of the Aeronautical Sciences, Hamburg, Germany, September 2006

10. Guenov, M.D., Utyuzhnikov, S.V., Fantini, P.: Application of the Modified Physical Programming Method to Generating the Entire Pareto Frontier in Multiobjective Optimization. In: Proceedings of EUROGEN 2005, Munich, Germany, September 2005

11. Maginot, J.: Sensitivity analysis for multidisciplinary design optimization. PhD Thesis, Cranfield University, Cranfield, UK (2007)

12. Maginot, J., Guenov, M.D., Fantini, P., Padulo, M.: A method for assisting the study of Pareto solutions in multi-objective optimization. In: Proceedings of the $7^{\text {th }}$ AIAA/ATIO Conference, Belfast, Northern Ireland, September 2007

13. Mattison, C.A., Mullur, A.A., Messac, A.: Minimal Representation of Multiobjective Design Space Using Smart Pareto Filter. In: Proceeding of the $9^{\text {th }}$ AIAA/ISSMO Symposium on Multidisciplinary Analysis and Optimization, Atlanta, GA, September 2002

14. Messac A., Mattson C.A.: Generating Well-Distributed Sets of Pareto Points for Engineering Design using Physical Programming. Optimization and Engineering, Kluwer Publishers, Vol. 3, Issue 4, pp. 431-450 (2002)

15. Messac, A., Ismail-Yahaya, A., Mattson, C.A.: The Normalized Normal Constraint Method for Generating the Pareto Frontier. Journal of the International Society of Structural and Multidisciplinary Optimization (ISSMO), Springer, Vol. 25, No. 2, pp. 86-98 (2003)

16. Messac, A., Mattson, C.: Normal Constraint Method with Guarantee of Even Representation of Complete Pareto Frontier. AIAA Journal, Vol. 42, No. 10, pp. 2101-2111 (2004)

17. Messac, A.: Physical Programming: Effective Optimization for Computational Design. AIAA Journal, Vol. 34, No. 1, pp. 149-158 (1996)

18. Miettinen, K.M.: Nonlinear Multiobjective Optimization. Kluwer Academic, Boston (1999)

19. Murphy, T.E., Tsui, K.L., Allen J.K.: A review of robust design methods for multiple responses. Research in Engineering Design, 16, pp.118-132 (2005)

20. Padulo, M., Campobasso, M.S., Guenov, M.D.: Comparative Analysis of Uncertainty Propagation methods for Robust Engineering Design. In: Proceedings of the International Conference on Engineering Design ICED07, Paris, August 2007

21. Park, G.J., Lee, T.H., Hwang, K.H.: Robust Design: An Overview. AIAA Journal, 44(1), pp. 181-191 (2006)

22. Saltelli, A., Chan, K., Scott, M.: Sensitivity Analysis. John Wiley \& Sons, New York (2000)

23. Saltelli, A., Tarantola, S., Campolongo, F., and Ratto, M.: Sensitivity Analysis in Practice: a guide to assessing scientific models. John Wiley \& Sons, Chichester (2004)

24. Saltelli, A., Bolado, R.: An alternative way to compute Fourier Amplitude Sensitivity Test (FAST). Computational Statistics \& Data Analysis, Vol. 26, No. 4, pp. 445-460 (1998)

25. Sobol', I.M.: Sensitivity Estimates for non-linear mathematical models. Mathematical Modelling \& Computational Experiments, Vol. 1, No. 4, pp. 407-414 (1993) 
26. Sobol', I.M.: Global sensitivity indices for non-linear mathematical models and their Monte Carlo estimates. Mathematics and Computers in Simulation, Vol. 55, pp. 271-280 (2001)

27. Utyuzhnikov, S.V., Fantini, P., Guenov, M.D.: Numerical Method for Generating the Entire Pareto Frontier in Multiobjective Optimization. In: Proceedings of EUROGEN 2005, 12-14, Munich, Germany, September 2005 